\title{
Discrepancia Dentaria de Bolton y Finalización de Ortodoncia: Consideraciones Clínicas
}

\author{
Bolton Tooth Size Discrepancy and Orthodontics Finalization: Clinical Considerations \\ Rogério Lacerda dos Santos ${ }^{*} \&$ Matheus Melo Pithon ${ }^{* *}$
}

\begin{abstract}
DOS SANTOS, L. R. \& PITHON, M. M. Discrepancia dentaria de Bolton y finalización de ortodoncia: consideraciones clínicas. Int. J. Odontostomat., 4(1):93-100, 2010.

RESUMEN: Las discrepancias entre el tamaño mesiodistal de los dientes superiores e inferiores, así como sus efectos sobre la oclusión han sido reportadas desde mediados del siglo XX. El objetivo de este estudio fue determinar la importancia de la aplicación del análisis de discrepancias dentaria de Bolton con finalidad ortodontica. Fueron seleccionados casos de pacientes de raza blanca de ambos sexos, con edades comprendidas entre 12 y 25 años. El análisis de las discrepancias dentarias fue realizado en la fase previa al tratamiento y posterior a la contención. Como criterio básico para la inclusión en la muestra de pacientes en el pre-tratamiento deberían presentar todos los dientes permanentes (de primeros molares a primeros molares del lado opuesto, en ambos arcos). Fueron eliminados los casos que presentaron dientes con grandes destrucciones, restauraciones inadecuadas y dientes mal posicionados donde no fue posible la medición adecuada entre los puntos de contacto. La medición del tamaño mesiodistal de los dientes se realizó con un caliper (Odin, Alemania), precisión de $0,01 \mathrm{~mm}$, por un solo examinador, previamente calibrado para realizar este procedimiento. Se midió el diámetro mesiodistal mayor de todos los dientes. Las relaciones totales y anteriores fueron calculadas por las fórmulas respectivas, según lo propuesto por Bolton (1958). Al finalizar, los casos mostraron que una discrepancia dentaria de Bolton maxilar o mandibular por exceso o falta dentaria, puede ser compensada por la alteración en la forma del arco, dimensión vestibulolingual y por la inclinación axial de los dientes anteriores, utilizadas en conjunto o aisladamente en la arcada, sin perjuicio estético y funcional.
\end{abstract}

PALABRAS CLAVE: ortodoncia, discrepancia dentaria, maloclusión.

\section{INTRODUCCIÓN}

La era actual ha tenido un dramático progreso en el campo de la ortodoncia clínica. Esto es particularmente cierto con respecto a la fase mecánica de tratamiento. Los últimos esfuerzos de investigación se han hecho para aclarar y simplificar el tratamiento, pero a menudo no se presta la debida atención a la finalización de la ortodoncia, ignorando un fundamento básico durante este, como son las discrepancias dentarias.

Las discrepancias entre el tamaño mesiodistal de los dientes superiores e inferiores, así como sus efectos sobre la oclusión en la finalización de la ortodoncia, se han reportado desde mediados del siglo XX. Varios autores han argumentado que discrepancias dentarias individuales o en grupos de dientes podrían estar asociadas con la aparición diastemas o apiñamientos, ausencia de intercuspidación, modificaciones en el resalte, sobremordida y la curva de Spee (Bolton, 1958, 1962; Tayer, 1992; Heusdens et al., 2000; Smith et al., 2000).

Algunos estudios realizados a partir del análisis de la oclusión normal, propusieron proporciones ideales entre el tamaño de los dientes superiores e inferiores (Ballard, 1944; Bolton, 1958, 1962; Barrett et al., 1963; Hixon \& Oldfather, 1958; Hunter \& Priest, 1960; Lundstron, 1981; Moorres \& Reed, 1994; Nance, 1947; Neff, 1949; Peck \& Peck, 1972; Saatçi \& Yukay, 1997), siendo que el método propuesto por Bolton (1958), se convirtió en uno de los más difundidos y aceptados, principalmente por tratarse de un análisis de fácil rea-

* Especialista em Ortodontia pela Universidade Federal de Alfenas - UNIFAL, Mestre e Doutorando em Ortodontia pela Universidade Federal do Rio de Janeiro - UFRJ. Brasil.

** Especialista em Ortodontia pela Universidade Federal de Alfenas - UNIFAL, Mestre e Doutorando em Ortodontia pela Universidade Federal do Rio de Janeiro - UFRJ. Brasil. 
lización y aplicación en los tratamiento ortodónticos (Smith et al.; Saatçi \& Yukay; Nie \& Lin, 1999; Ta et al., 2001).

Otros estudios han evaluado la relación entre dimorfismo sexual en el tamaño y proporciones de los dientes (Smith et al.; Nie \& Lin; Ta et al.; Woodworth et al., 1985; Carreiro, 2005), sin embargo, existen estudios que no consideran esta variable en el análisis de la desarmonía del tamaño dentario (Crosby \& Alexander, 1989; Freeman et al., 1996; Brunharo et al., 2002). El tipo de maloclusión es un factor que debe ser considerarado en la muestra según algunos autores (Nie \& Lin; Ta et al.; Carreiro; Freeman et al.), aunque otros estudios no consideran esto en la caracterización de los pacientes (Smith et al.; Freeman et al.; Brunharo et al.). No existe consenso sobre la necesidad de incluir estos factores en la construcción de la muestra.

La influencia de la etnia en las dimensiones y proporciones dentarias fue señalado por algunos autores (Smith et al.; Nie \& Lin; Ta et al.) para dividir la muestra. En Brasil, el establecimiento del origen preciso de los pacientes es difícil, ya que existe una amplia mezcla racial de la población (Motta et al., 2008).

Shellhart et al. (1995) evaluaron la confiabilidad del análisis de Bolton cuando el arco presentaba apiñamiento, concluyendo que la presencia de dientes mayores a $3 \mathrm{~mm}$ hacen que la evaluación sea menos precisa. Halazonetis (1996) propuso una forma simple de evaluar el análisis de Bolton. Sus resultados mostraron que la forma del arco, así como el diámetro vestibulolingual de los dientes anteriores pueden exigir correcciones, particularmente en los dientes anteriores. Variaciones de $1 \mathrm{~mm}$ en el grosor del borde incisal (vestibulolingual) puede alterar en un $5 \%$ el índice, es decir, una diferencia de casi $3 \mathrm{~mm}$ en los dientes anteriores, donde $1 \mathrm{~mm}$ de resalte puede ser compensado por el exceso de 1 a $3 \mathrm{~mm}$ en el diámetro de los bordes incisales, dependiendo de la curvatura del arco. Por lo tanto, en el caso de compensación dentaria, además de las inclinaciones dentarias, una eventual discrepancia de bases óseas puede estar enmascarada variando la forma del arco y el diámetro vestibulolingual de los bordes incisales.

También es importante señalar que además de la suma de los tamaños dentários, el grado de sobremordida y el resalte, también puede estar influenciado por la inclinación de los incisivos, así como la relación de las bases apicales. Por lo tanto, si el paciente presenta distorsiones en la posición de los dientes y/o bases apicales, no se puede correlacionar el resalte y la sobremordida de manera directa con los tamaños dentarios (Ramos et al., 1997).

La tabla de valores ideales para los dientes superiores e inferiores provienen de individuos con oclusión normal, por lo tanto, no sufren la influencia de estas dos variantes mencionadas anteriormente. Esto es particularmente importante en los casos donde el tratamiento de ortodoncia conlleva una compensación dentaria (por inclinación) para enmascarar una leve discrepancia de bases óseas. Así, incluso si el análisis de Bolton se realiza al inicio del tratamiento y evidencia exceso de masa dentaria inferior, por ejemplo, nos indicarian desgastes obligatorios si una mandíbula se presenta en retroposición, porque para la compensación los incisivos serían proyectados y compensaria el exceso de masa dentaria.

Estudios de Crosby \& Alexander y Freeman et al. encontraron incidencias de 23 y $30 \%$ respectivamente, de pacientes con discrepancias en el tamaño entre los arcos dentales, mayores a 2 desviaciones estándar, lo que justifica la recomendación de los autores de la realización rutinaria del análisis de Bolton.

Dado lo anterior, este estudio tiene por objetivo analizar la importancia de la aplicabilidad del análisis de discrepancia dentaria de Bolton al finalizar el tratamiento de ortodoncia. Se discutirá a partir de 4 modelos de casos de tratamientos de ortodoncia finalizada de la Clínica del Programa de Post-Graduación en Ortodoncia de la Universidad Federal de Río de Janeiro UFRJ. Todos los pacientes firmaron un consentimiento informado para la divulgación de la documentación de ortodoncia.

\section{RELATO DE CASOS}

\section{Caso 1}

Maloclusión: CLII, $2^{\circ}$ división, subdivisión izquierda Bolton: $\Sigma$ Total inferior: $90,5 \mathrm{~mm}$

$\sum$ Total superior: $98,5 \mathrm{~mm}$

$\Sigma$ = suma de tamaño M-D

Índice total: 91,8 (sin exceso)

$\sum$ Anterior inferior: $39,5 \mathrm{~mm}$

$\sum$ Anterior superior: $50,25 \mathrm{~mm}$

Îndice anterior: 78,6 (exceso inferior 1,0 mm)

Características dentárias iniciales (Figs. $1 \mathrm{~A}-\mathrm{E}$ ):

Overjet: $4 \mathrm{~mm}$ 
Overbite: $80 \%$

Discrepancia de modelo inferior: $-1 \mathrm{~mm}$

Forma de arco inferior: parabólico

Forma de arco superior: parabólico

Características dentárias finales (Figs. 2 A-E):

Maloclusión: Llave de oclusión de 1er molar y canino

Overjet: $1 \mathrm{~mm}$
Overbite: $20 \%$

Forma de arco inferior: parabólico

Forma de arco superior: parabólico

Consideraciones clínicas: fueron utilizadas compensaciones en las inclinaciones axiales mesiodistales de los incisivos superiores ("artistic bend") para compensar el exceso dentario inferior.
Fig. 1A. Modelo inicial articulado (vista frontal).

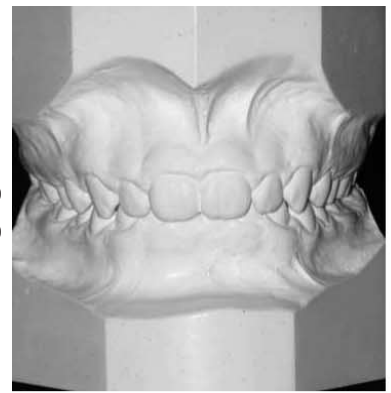

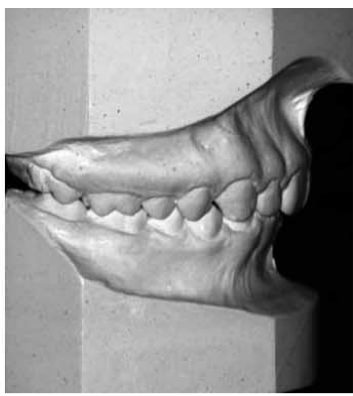

Fig. 1B. Modelo inicial articulado (vista lateral derecha).

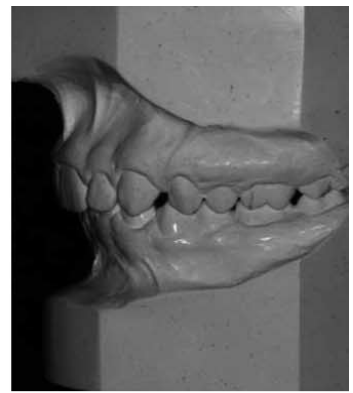

Fig. 1C. Modelo inicial articulado (vista lateral izquierda).
Fig. 1D. Modelo inicial inferior (vista oclusal inferior).
Fig. 2A. Modelo final articulado (vista frontal).

Fig. 2D. Modelo final inferior (vista oclusal inferior).
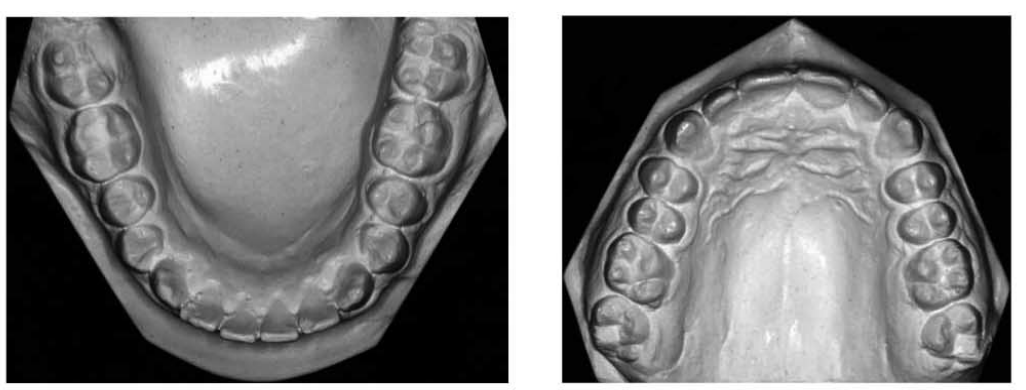

Fig. 1E Modelo inicial superior (vista oclusal superior)

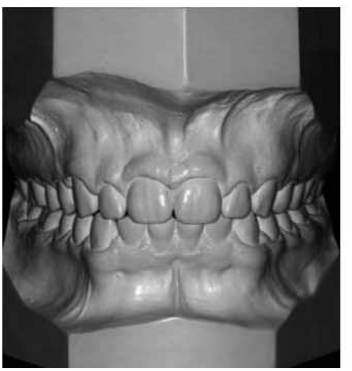

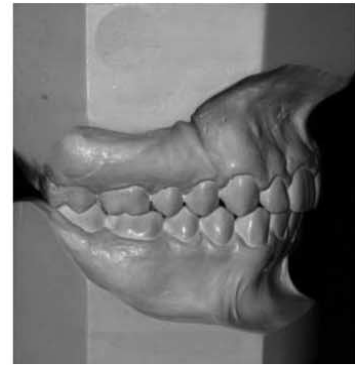

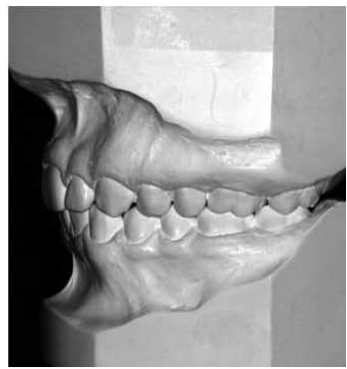

Fig. 2C. Modelo final articulado (vista lateral esquerda).

Fig. 2B. Modelo final articulado (vista lateral direita).
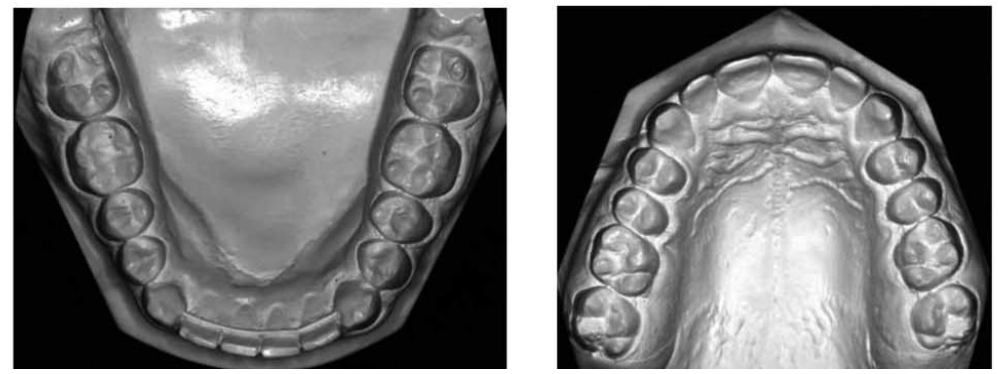

Fig. 2E. Modelo final superior (vista oclusal superior). 


\section{Caso 2}

Maloclusión: CLII, $1^{\circ}$ división, subdivisión derecha Bolton: $\sum$ Total inferior: $84,0 \mathrm{~mm}$

$\sum$ Total superior: $97,0 \mathrm{~mm}$

Índice total: 86,50 (exceso superior $5,0 \mathrm{~mm}$ )

$\sum$ Anterior inferior: $36,5 \mathrm{~mm}$

$\sum$ Anterior superior: $48,5 \mathrm{~mm}$

Índice anterior: 75,25 (exceso superior 1,0mm)

Características dentárias iniciales (figuras $3 \mathrm{~A}-\mathrm{E}$ ):

Overjet: $5 \mathrm{~mm}$

Overbite: $70 \%$

Discrepancia de modelo inferior: $-1,5 \mathrm{~mm}$

Forma de arco inferior: en forma de "V" (contraído)
Forma de arco superior: en forma de "V" (contraído)

Características dentárias finales (figuras $4 \mathrm{~A}-\mathrm{E}$ ):

Maloclusión: Llave de oclusión de 1er molar y canino Overjet: $1 \mathrm{~mm}$

Overbite: $30 \%$

Forma de arco inferior: en forma de "V"

Forma de arco superior: en forma de "V"

Consideraciones clínicas: fueron realizados desgastes mesiodistales y verticalización axiomesio-distal (ausencia de "artistic bend") en los incisivos superiores, distalización de los molares superiores asociado a expansión del arco superior y verticalización de los dientes posteriores e inferiores mejorando la forma del arco para compensar exceso superior.
Fig. 3A. Modelo inicial articulado (vista frontal).

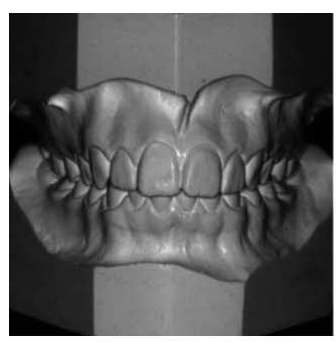

Fig. 3D. Modelo inicial inferior (vista oclusal inferior).

Fig. 4A. Modelo final articulado (vista frontal).

Fig. 4D. Modelo final inferior (vista oclusal inferior).
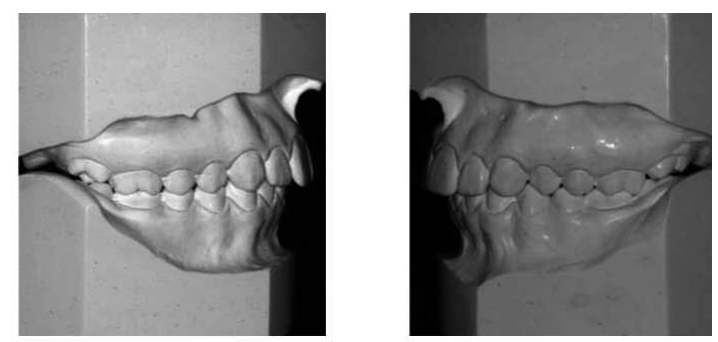

Fig. 3B. Modelo inicial articulado (vista lateral derecha)
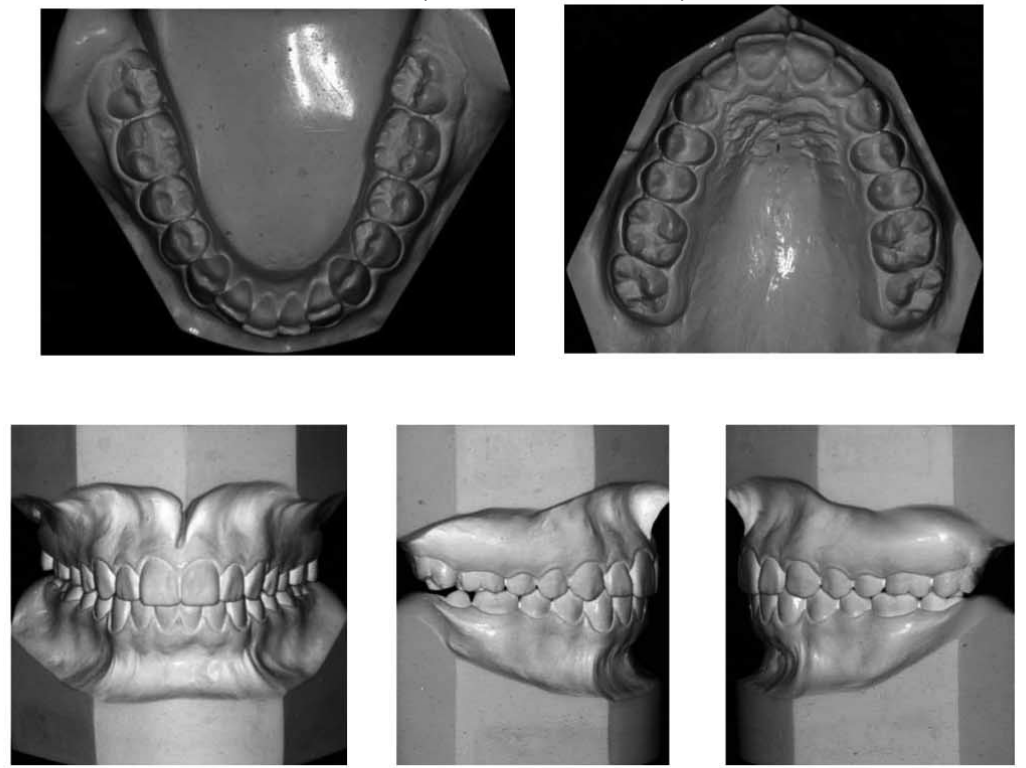

Fig. 4B. Modelo final articulado (vista lateral derecha).
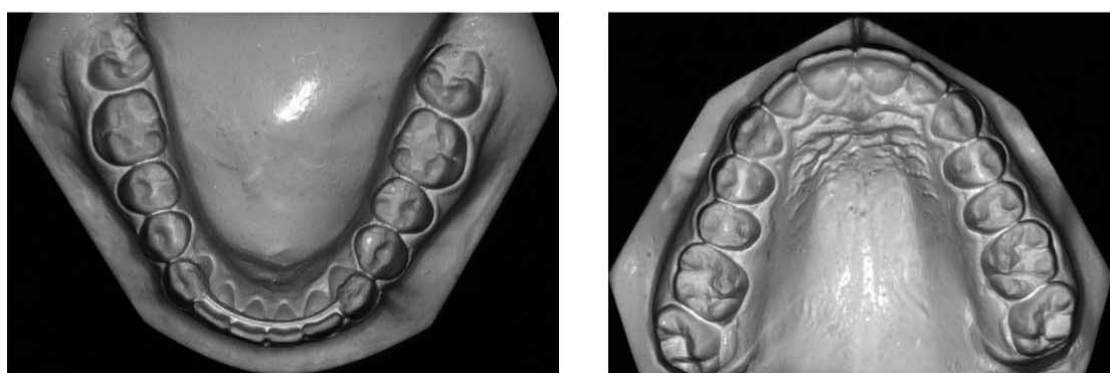

Fig. 3C. Modelo inicial articulado (vista lateral esquerda)
Fig. 3E. Modelo inicial superior (vista oclusal superior)

Fig. 4C. Modelo final articulado (vista lateral esquerda)
Fig. 4E. Modelo final superior (vista oclusal superior) 


\section{Caso 3}

Maloclusión: CLI

Bolton: $\Sigma$ Total inferior: $90,0 \mathrm{~mm}$

$\sum$ Total superior: $97,5 \mathrm{~mm}$

Índice total: 92,3 (exceso inferior 1,5 mm)

$\sum$ Anterior inferior: $37,5 \mathrm{~mm}$

$\sum$ Anterior superior: $47,5 \mathrm{~mm}$

Índice anterior: 78,9 (exceso inferior 0,8 mm)

Características dentárias iniciales (figuras $5 \mathrm{~A}-\mathrm{E}$ ):

Overjet: $2 \mathrm{~mm}$

Overbite: mordida abierta (4 $\mathrm{mm})$

Discrepáncia de modelo inferior: $2,5 \mathrm{~mm}$
Forma de arco inferior: parabólico

Forma de arco superior: en forma de "V"

Características dentárias finales (figuras 6 A-E):

Maloclusión: Llave de oclusión de 1er molar y canino

Overjet: $1 \mathrm{~mm}$

Overbite: $30 \%$

Forma de arco inferior: parabólico

Forma de arco superior: parabólico

Consideraciones clínicas: La compensación de exceso inferior fue realizada a costa del posicionamiento axial correto de los dientes en el arco superior, projección e inclinación axial mesiodistal ("artistic bend") de los incisivos superiores.
Fig. 5A. Modelo inicial articulado (vista frontal).

Fig. 5D. Modelo inicial inferior (vista oclusal inferior)
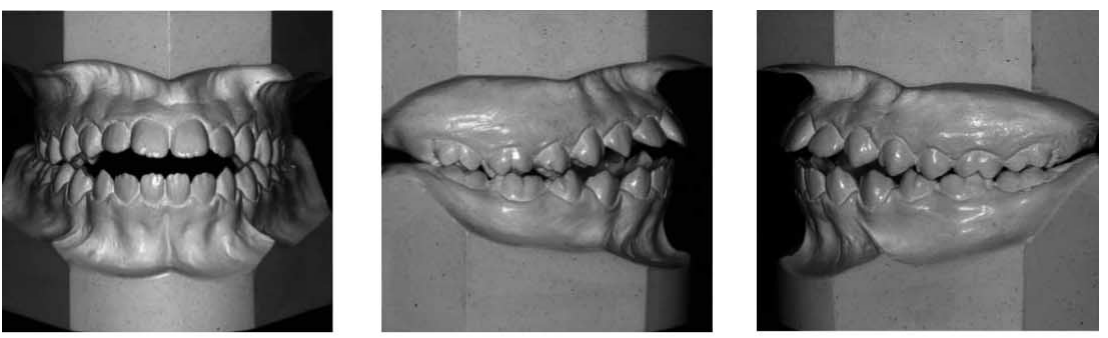

Fig. 5C. Modelo inicial articulado (vista lateral izquierda).
Fig. 5B. Modelo inicial articulado (vista lateral derecha)
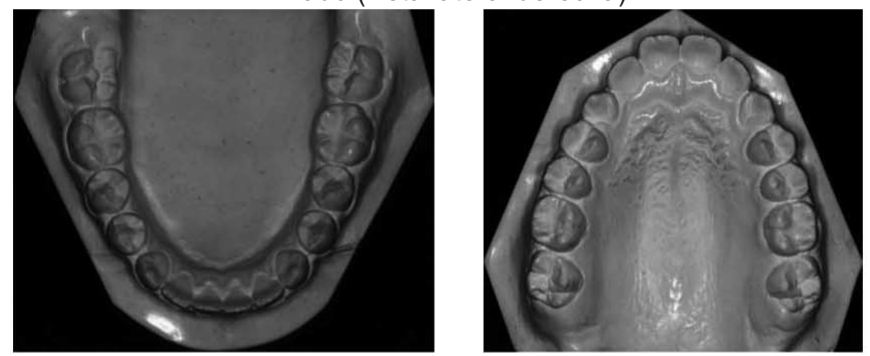

Fig. 5E. Modelo inicial superior (vista oclusal superior).
Fig. 6A. Modelo final articulado (vista frontal).
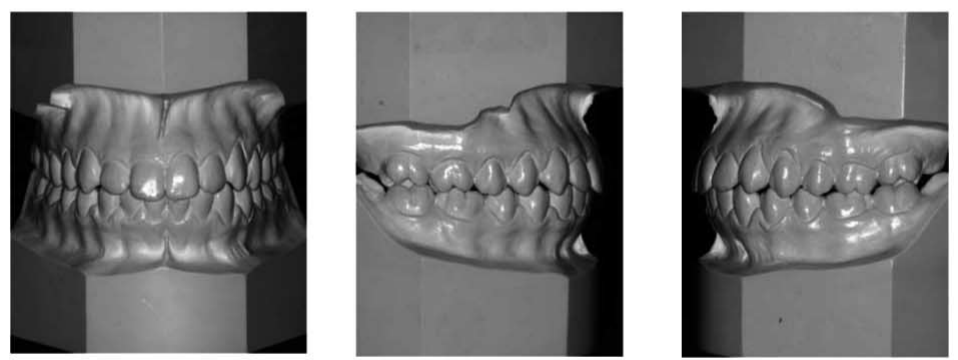

Fig. 6B. Modelo final articulado (vista lateral derecha).
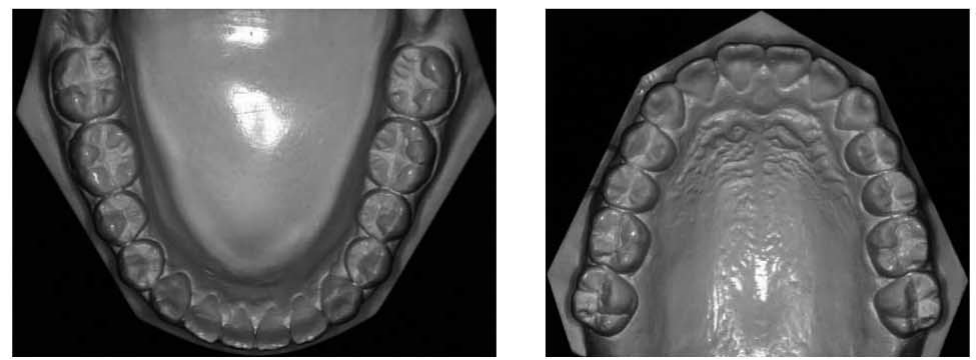

Fig. 6C. Modelo final articulado (vista lateral izquierda).

Fig. 6E. Modelo final superior (vista oclusal superior) 


\section{Caso 4}

Maloclusión: CLII, $1^{\circ}$ división

Bolton: $\sum$ Total inferior: $93,5 \mathrm{~mm}$

$\sum$ Total superior: $104,1 \mathrm{~mm}$

Índice total: 89,8 (exceso superior 2,0 mm)

$\sum$ Anterior inferior: $39,5 \mathrm{~mm}$

$\sum$ Anterior superior: $52,2 \mathrm{~mm}$

Índice anterior: 75,6 (exceso superior 1,2 mm)

Características dentárias iniciales (figuras 7 A-E):

Overjet: $6 \mathrm{~mm}$

Overbite: $80 \%$
Discrepancia de modelo inferior: ausente

Forma de arco inferior: parabólico

Forma de arco superior: en forma de "V"

Características dentarias finales (figuras $8 \mathrm{~A}-\mathrm{E}$ ):

Maloclusión: Llave de oclusión de 1er molar y canino

Overjet: $1 \mathrm{~mm}$

Overbite: $20 \%$

Forma de arco inferior: parabólico

Forma de arco superior: parabólico

Consideraciones clínicas: Fueron realizados desgastes mesiodistales y verticalización axiomesiodistal (auséncia de "artistic bend") en los incisivos superiores y expansión de arco superior.
Fig. 7A. Modelo inicial articulado (vista frontal)
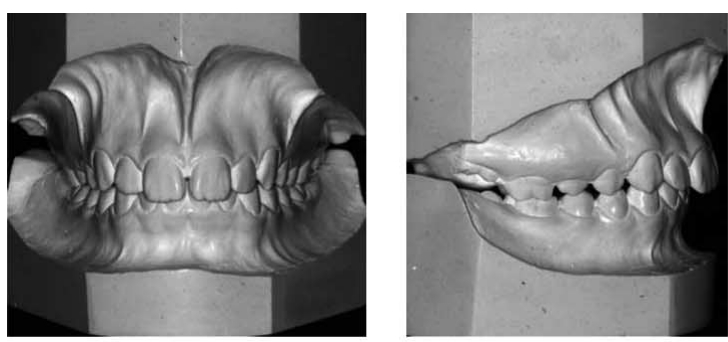

Fig. 7B. Modelo inicial articulado (vista lateral derecha).

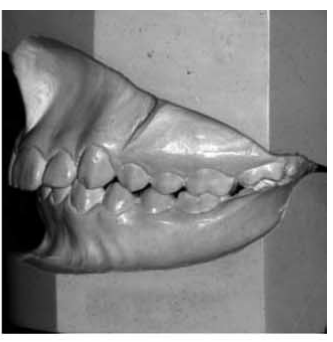

Fig. 7C. Modelo inicial articulado (vista lateral izquierda).

Fig. 7D. Modelo inicial inferior (vista oclusal inferior).

Fig. 8A. Modelo final articulado (vista frontal).

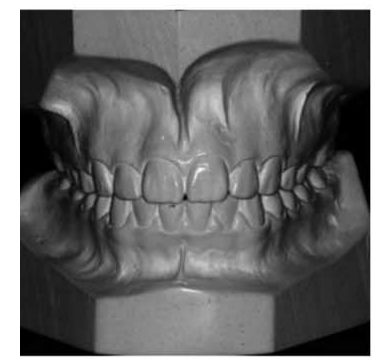

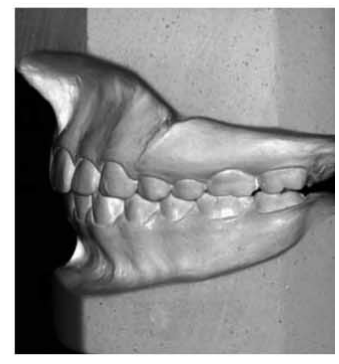

Fig. 8B. Modelo final articulado (vista lateral derecha).
Fig. 8C. Modelo final articulado (vista lateral izquierda).
Fig. 8D. Modelo final inferior (vista oclusal inferior).
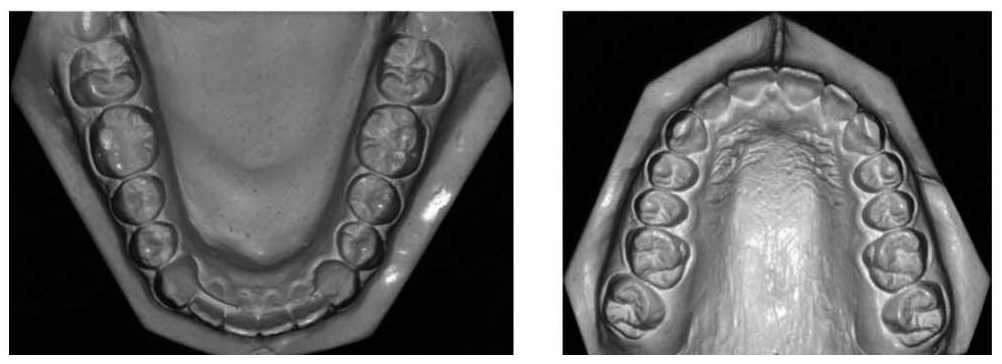

Fig. 7E. Modelo inicial superior (vista oclusal superior).
Fig. 8E. Modelo final superior (vista oclusal superior). 


\section{DISCUSIÓN}

Shellhart et al. evaluaron la confiabilidad del análisis de Bolton en el arco dentario con apiñamiento, concluyendo que la presencia de un apiñamiento mayor a $3 \mathrm{~mm}$ hace que la evaluación sea menos precisa. Ninguno de los casos descritos en nuestro trabajo presentó un apiñamiento mayor a $3 \mathrm{~mm}$, es de destacar que grandes apiñamientos pueden generar análisis erróneos, ya que puede existir dificultad en la medición de los diámetros mesiodistales de los dientes.

El tamaño dentario excesivo puede ser compensado por los cambios en el arco (Halazonetis), como se observa en los casos 2, 3 y 4 en donde la expansión transversal, o incluso la corrección de las inclinaciones axiales vestibulolinguales de los arcos mejoraron la intercuspidación.

La discrepancia dentaria individual o en los grupos de dientes puede estar asociada con la aparición de diastemas o apiñamientos, ausencia de intercuspidación, modificasiones en el resalte y la sobremordida (Bolton, 1958, 1962; Heusdens et al.; Smith et al.; Tayer), donde estas discrepancias pueden ser corregidas a través del desgaste proximal como en los casos 2 y 4 o a traves de compensaciones axiales, como inclinaciones mesiodistales o vestibulolinguales. Las compensaciones axiales como "artistic bend" en los incisivos superiores pueden resolver pequeñas discrepancias de exceso dentario mandibular como en los casos 1 y 3 , o la falta de "artistic" como en los casos 2 y 4 pueden estar asociadas con disminución de overjet, retroinclinación de los incisivos inferiores y vestibularización de los incisivos superiores en casos que presentan discrepancias mayores de exceso mandibular, como en el caso 3.
La distalización de los dientes posteriores también pueden ayudar en la corrección de las discrepancias dentales en el caso 2, pero el exceso de distalización en los casos que presentan discrepancias mayores, pueden significar un perjuicio para la intercuspidación.

La dimensión vestíbulolingual de los incisivos puede generar variaciones capaces de interferir en la finalización del tratamiento (Bolton, 1958, 1962; Heusdens et al.; Smith et al.; Tayer), pero es más pronunciada en los incisivos que tienen alteraciones de forma y tamaño, requiriendo correcciones, y cuando el desgaste dentario es propuesto, este debe ser realizado después de evaluar la existencia de esmalte proximal suficiente para permitir la integridad dentaria.

Durante la finalización del tratamiento ortodóntico es necesario verificar cada caso, teniendo como meta la llave de caninos, respetando los movimientos funcionales de la mandíbula para la correcta orientación axial entre los caninos superiores e inferiores, además de la centralización de las líneas medianas dentaria y facial.

La diferencia de tamaño dentario maxilar o mandibular por exceso o falta puede ser compensada por la alteración en la forma del arco, dimensión mesiodistal y por las inclinaciones axiales de los dientes anteriores, utilizados en conjunto o por separado en la arcada, sin un perjuicio estético y funcional. Además, se sugieren restauraciones estéticas en casos de discrepancias marcadas por falta dentaria. Finalmente, la alineación y nivelación de los dientes y la correcta intercuspidación diente a dos dientes son requisitos previos para la evaluación estática de los pacientes al finalizar su tratamiento de ortodoncia.

DOS SANTOS, L. R. \& PITHON, M. M. Bolton tooth size discrepancy and orthodontics finalization: Clinical considerations.Int. J. Odontostomat., 4(1):93-100, 2010.

ABSTRACT: Discrepancies of the mesiodistal dimensions between upper and lower teeth as well as their effects on occlusion have been reported since the middle of the twentieth century. The aim of this study was to elucidate the importance of the applicability of the analysis of the Bolton tooth size discrepancy in the orthodontic finalizing. Cases involving Caucasian male and female patients aged between 12 and 25 years were selected for study. Analyses of the dental discrepancies were carried out before orthodontic treatment and after contention. Basically, only patients having all permanent teeth before treatment (with first molars in both arches) were included. Those cases of severely mutilated teeth, inadequate restorations, and tooth malalignment were ruled out because accurate measurement of the contacting points could not be performed. Measurement of the mesiodistal tooth dimension was performed by only one operator using a digital calliper of a resolution of $0.001 \mathrm{~mm}$ (Odin, Germany) which had been previously calibrated for such procedures. It was measured the largest mesiodistal diameter. Both previous and total relationships were calculated through their respective formulas according to Bolton (1958). The orthodontic finalisation showed that Bolton tooth size discrepancies involving either crowded dentition or lack of teeth can be compensated by mandibular or maxillary arch shape, buccal-lingual dimension, and axial inclination of the anterior teeth with no aesthetic or functional impairment. 


\section{REFERENCIAS BIBLIOGRÁFICAS}

Ballard, M. L. Asymmetry in tooth size: A factor in the etiology, diagnosis and treatment of malocclusion. Angle Orthod., 14:65-70, 1944.

Barrett, M. J.; Brown, T. \& MacDonald, M. R. Dental observations on the Australian Aborigines: Mesio-distal crown diameter of the permanent teeth. Aust. Dent. J., 8:150-5, 1963.

Bolton, W. A. The clinical application of a tooth-size analysis. Am. J. Orthod. Dentofacial Orthop., 48:504-29, 1962.

Bolton, W. A. Disharmony in tooth size and its relation to the analysis and treatment of malocclusion. Angle Orthod., 28:113-30, 1958.

Brunharo, I. H. V. P.; Almeida, M. A. O.; Tibana, R. H. W.; Guido, A. T. F. \& Paula, S. Avaliação comparativa entre dois métodos de medição do diâmetro dentário. R. Dental Press Ortodon. Ortop. Facial, Maringá, 7:51-5, 2002.

Carreiro, L. S. A discrepância de tamanho dentário, de Bolton, na oclusão normal e nos diferentes tipos de más oclusões, bem como sua relação com a forma de arco e o posicionamento dentário. R. Dental Press Ortodon. Ortop. Facial, Maringá, 10:97-117, 2005.

Crosby, D. A. \& Alexander, C. G. The occurrence of tooth size discrepancies among different malocclusion groups. Am. J. Orthod. Dentofacial Orthop., 95:457-61, 1989.

Freeman, J. E.; Maskeroni, A. J. \& Lorton, L. Frequence of Bolton tooth-size discrepancies among orthodontic patients. Am. J. Orthod. Dentofacial Orthop., 110:24-7, 1996.

Halazonetis, D. J. The Bolton ratio studied with the use of spreadsheets. Am. J. Orthod. Dentofacial Orthop., 102:2159, 1996.

Heusdens, M.; Dermaut, L. \& Verbeeck, R. The effect of tooth size discrepancy on occlusion: An experimental study. Am. J. Orthod. Dentofacial Orthop., 117:184-91, 2000.

Hixon, E. H. \& Oldfather, R. E. Estimation of the sizes of unerupted cuspid and bicuspid teeth. Angle Orthod., 28:236-40, 1958.

Hunter, W. S. \& Priest, W. R. Errors and discrepancies in measurement of tooth size. J. Dent. Res., 39:405-14, 1960.

Lundstron, A. Intermaxillary tooth width ratio analysis. Eur. J. Orthod., 3:285-87, 1981.

Moorres, C. F. A. \& Reed, R. B. Biometrics of crowding and spacing of the teeth in the mandible. Am. J. Phys.
Anthropol., 12:77-88, 1994.

Motta, A. T. S.; Rodrigues, S.; Quintão, C. C. A. \& Capelli, J. J. Análise da discrepância de tamanho dentário em pacientes da Clínica de Ortodontia da FO/UERJ. R. Dental Press Ortodont. Ortop. Facial, Maringá, 9:83-90, 2008.

Nance, H. N. Limitations of Orthodontic treatment. Part I. Am. J. Orthod., 33:253-301, 1947.

Neff, C. W. Tailored occlusion with the anterior coefficient. Am. J. Orthod., 35:309-13, 1949.

Nie, Q. \& Lin, J. Comparasion of intermaxillary tooth size discrepancies among different malocclusion groups. Am. J. Orthod. Dentofacial Orthop., 116:539-44, 1999.

Peck, H. \& Peck, S. A Index for assessing tooth shape deviations as applied to the mandibular incisors. Am. J. Orthod., 61:384-401, 1972.

Ramos, L. A.; Suguino, R.; Terada, H. H.; Furquim, Z. L. Filho, S. O. G. Considerações sobre análise da discrepância dentária de Bolton e a finalização ortodôntica. R. Dental Press Ortodon. Ortop. Facial, Maringá, 1:86-106, 1996.

Saatçi, P. \& Yukay, T. H. The effect of premolar extractions on tooth-size discrepancy. Am. J. Orthod. Dentofacial Orthop., 111:428-34, 1997.

Shellhart, W. C.; Lange, D. W.; Kluemper, G. T.; Hicks, E. P. \& Kaplan, A. L. Reability of the Bolton tooth-size analysis when applied to crowded dentitions. Angle Orthod., 65:32734, 1995.

Smith, S. S.; Buschang, P. H. \& Watanabe, E. Interarch tooth size relationships of 3 populations: "Does Bolton's analysis apply?". Am. J. Orthod. Dentofacial Orthop., 117:169-74, 2000.

Ta, T. A.; Ling, J. Y. K. \& Hagg, U. Tooth-size discrepancies among different occlusion groups of southern Chinese children. Am. J. Orthod. Dentofacial Orthop., 120:556-8, 2001.

Tayer, B. H. The asymmetric extraction decision. Angle Orthod., 62:291-7, 1992.

Woodworth, D. A.; Sinclair, P. M. \& Alexander, R. G. Bilateral congenital absence of maxillary lateral incisors: a craniofacial and dental cast analysis. Am. J. Orthod., 87:280-93, 1985.

Dirección para Correspondencia:

Rogério Lacerda dos Santos

Praça José Batista de Freitas, 78, sala 102, centro

Cep:35.519.000 Nova Serrana-MG

BRASIL

e-mail:lacedaorto@hotmail.com

Recibido : 31-08-2009

Aceptado: 18-01-2010 